\title{
On Truncated Variation of Brownian Motion with Drift by
}

\section{Rafał ŁOCHOWSKI}

\author{
Presented by Stanisław KWAPIEŃ
}

Summary. We introduce the concept of truncated variation of Brownian motion with drift, which differs from regular variation by neglecting small jumps (smaller than some $c>0$ ). We estimate the expected value of the truncated variation. The behaviour resembling phase transition as $c$ varies is revealed. Truncated variation appears in the formula for an upper bound for return from any trading based on a single asset with flat commission.

1. Introduction. Let $\left(W_{t}, t \geq 0\right)$ be a Wiener process on the interval $[0, T]$ with drift $\mu, W_{t}=\mu t+B_{t}$, where $\left(B_{t}, t \geq 0\right)$ is a standard Brownian motion.

It is well known (cf. [4]) that for any $a<b$, the variation of this process on $[a, b]$ is infinite:

$$
\sup _{n} \sup _{a \leq t_{1}<\cdots<t_{n} \leq b} \sum_{i=1}^{n-1}\left|W_{t_{i+1}}-W_{t_{i}}\right|=+\infty .
$$

However, if we restrict ourselves to jumps greater than some $c>0$ and define the truncated variation of $\left(W_{t}, t \geq 0\right)$ on $[a, b], V_{\mu}^{c}[a, b]$, as

$$
V_{\mu}^{c}[a, b]=\sup _{n} \sup _{a \leq t_{1}<\cdots<t_{n} \leq b} \sum_{i=1}^{n-1} \max \left\{\left|W_{t_{i+1}}-W_{t_{i}}\right|-c, 0\right\},
$$

then we obtain a random variable which is finite almost surely. (A technical remark: for $a>b$ we put $V_{\mu}^{c, p}[a, b]=0$.) Truncated variation is shift invariant, i.e. for $0 \leq a<b, V_{\mu}^{c}[a, b]$ has the same distribution as $V_{\mu}^{c}[0, b-a]$. 
However, this functional is no longer additive, i.e. for $0 \leq a<b<d$ we do not have a.s. the equality

$$
V_{\mu}^{c}[a, d]=V_{\mu}^{c}[a, b]+V_{\mu}^{c}[b, d] .
$$

In this paper we will estimate, up to universal constants, the expected value of $V_{\mu}^{c}[0, T]$, i.e. we will find a function of parameters $\mu, c$ and $T$, $F(\mu, c, T)$, such that the ratio $\mathbb{E} V_{\mu}^{c}[0, T] / F(\mu, c, T)$ is separated from 0 and infinity. We give some numerical constants for this separation, but we do not attempt to obtain the best possible ones.

Since the truncated variation has the same value for the process $\left(W_{t}\right.$, $t \geq 0)$ as for the process $\left(-W_{t}, t \geq 0\right)$, we will assume $\mu \geq 0$. Let us also define

$$
\chi(c, \mu)=\sqrt{\frac{e^{2 \mu c}-1-2 \mu c}{2 \mu^{2}}}=c \sqrt{1+\frac{2}{3} \mu c+\cdots} .
$$

The function $F$ has the form

$$
F(\mu, c, T)= \begin{cases}T / c+\mu T & \text { if } \sqrt{T} \geq \chi(c, \mu) ; \\ 2 \sqrt{T}+\mu T-c & \text { if } c-\mu T \leq \sqrt{T}<\chi(c, \mu) ; \\ T^{3 / 2} \frac{\exp \left(-(c-\mu T)^{2} /(2 T)\right)}{(c-\mu T)^{2}} & \text { if } \sqrt{T} \leq c-\mu T .\end{cases}
$$

If we notice that $\chi(c, \mu)$ is of order $c$ when $\mu c \leq 1$ and of order $e^{\mu c} / \mu$ when $\mu c \geq 1$ we get even simpler formulae than above.

Thus $\mathbb{E} V_{\mu}^{c}[0, T]$ reveals some interesting behaviour. It is approximately linear in $T$ for large $T$ but decreases rapidly for small $T$. Small changes of $c$ may also lead to dramatic changes of $V_{\mu}^{c}[0, T]$.

Truncated variation appears naturally when profit from a trading strategy based on a single asset is considered in the presence of transaction costs. If the dynamics of the prices of the asset, $P_{t}$, is a geometric Brownian motion process, $P_{t}=\exp \left(\mu t+\sigma B_{t}\right)$, and the cost of every transaction dealing with this asset is proportional to the value of the transaction (flat commission), then the highest possible rate of return from any trading of this single asset during the time interval $[0, T]$ is bounded from above by $\exp \left(\sigma V_{\mu / \sigma}^{c / \sigma}([0, T])\right)-1$ with $c=\ln \frac{1+\gamma}{1-\gamma}$, where $\gamma$ is the fraction of the transaction value paid for commission.

The paper is organized as follows. In the next section estimates of the expected value of the truncated variation for long time intervals are presented and in the last section we deal with short time intervals. In the appendix we explain how truncated variation appears in the upper bound for return from trading a single asset in the geometric Brownian motion model. 


\section{Preparatory lemmas and estimates for long time intervals.} In order to estimate $\mathbb{E} V_{\mu}^{c}[a, b]$ we first define

$$
T_{c}=\inf \left\{t \geq 0: W_{t} \leq \sup _{0 \leq s \leq t} W_{s}-c\right\},
$$

i.e. $T_{c}$ is the first time the process $W_{t}$ drops below its maximum to date by $c$.

Let $T_{\text {sup }}^{c}$ be the last instant when the maximum of $W_{t}$ on $\left[0, T_{c}\right]$ is attained, and let $T_{\text {inf }}^{c} \leq T_{\text {sup }}^{c}$ be such that $W_{T_{\text {inf }}^{c}}=\inf _{0 \leq s \leq T_{\text {sup }}^{c}} W_{s}$.

In order to ease notation we put $(a)_{+}=\max \{a, 0\}$ for any real $a$.

Let us start with the following

Lemma 1. The random time $T_{c}$ is a stopping time which is a.s. finite, and

$$
V_{\mu}^{c}\left[0, T_{c}\right]=\left(W_{T_{\mathrm{sup}}^{c}}-W_{T_{\mathrm{inf}}^{c}}-c\right)_{+} .
$$

Proof. By results of Taylor (cf. [7]) we know that $\mathbb{E} T_{c}<\infty$, which immediately yields $T_{c}<\infty$ a.s.

Now we prove (2.1). Let $0 \leq t_{1}<\cdots<t_{l} \leq T_{c}$ be a partition of the random interval $\left[0, T_{c}\right]$. For any $1 \leq i \leq j \leq l$ we have $W_{t_{j}}-W_{t_{i}} \geq-c$, hence $\left(\left|W_{t_{i+1}}-W_{t_{i}}\right|-c\right)_{+}>0$ if and only if $W_{t_{i+1}}>W_{t_{i}}+c$. Let $j<k$ be two consecutive indices such that $W_{t_{j+1}}>W_{t_{j}}+c$ and $W_{t_{k+1}}>W_{t_{k}}+c$. Then

$$
\begin{aligned}
\left(\left|W_{t_{k+1}}-W_{t_{k}}\right|-c\right)_{+}+ & \left(\left|W_{t_{j+1}}-W_{t_{j}}\right|-c\right)_{+} \\
& =W_{t_{k+1}}-W_{t_{j}}-c-\left(W_{t_{k}}-W_{t_{j+1}}+c\right) \\
& \leq W_{t_{k+1}}-W_{t_{j}}-c=\left(\left|W_{t_{k+1}}-W_{t_{j}}\right|-c\right)_{+} .
\end{aligned}
$$

Iterating the above procedure we obtain

$$
\sum_{i=1}^{l-1}\left(\left|W_{t_{i+1}}-W_{t_{i}}\right|-c\right)_{+} \leq\left(W_{T_{\mathrm{sup}}^{c}}-W_{T_{\mathrm{inf}}^{c}}-c\right)_{+} .
$$

Taking the supremum over all partitions $0 \leq t_{1}<\cdots<t_{l} \leq T_{c}$ we get $V_{\mu}^{c}\left[0, T_{c}\right] \leq\left(W_{T_{\text {sup }}^{c}}-W_{T_{\text {inf }}^{c}}-c\right)_{+}$. Since the opposite inequality is obvious, we finally get $(2.1)$.

We also have

Lemma 2. The following inequalities hold:

$$
\begin{aligned}
V_{\mu}^{c}([0, T]) \leq & V_{\mu}^{c}\left[0, T_{c}\right]+c+V_{\mu}^{c}\left[T_{c}, T\right], \\
V_{\mu}^{c}([0, T]) \leq & V_{\mu}^{c}\left[0, T_{c}\right]+\left(W_{T_{\text {sup }}^{c}}-\inf _{T_{c} \leq t \leq T} W_{t}-c\right)_{+} \\
& +V_{\mu}^{c}\left[T_{c}, T\right] .
\end{aligned}
$$

Proof. Since for $T_{c} \geq T$ the inequalities (2.2) and (2.3) are self-evident, we will assume $T_{c}<T$. 
We will prove that for any partition $0 \leq t_{1}<\cdots<t_{n} \leq T$ of the interval $[0, T]$ and $S=\sum_{i=1}^{n-1}\left(\left|W_{t_{i+1}}-W_{t_{i}}\right|-c\right)_{+}$we have

$$
S \leq V_{\mu}^{c}\left[0, T_{c}\right]+c+V_{\mu}^{c}\left[T_{c}, T\right]
$$

and

$$
S \leq V_{\mu}^{c}\left[0, T_{c}\right]+\left(\left|W_{T_{\text {sup }}^{c}}-\inf _{T_{c} \leq t \leq T} W_{t}\right|-c\right)_{+}+V_{\mu}^{c}\left[T_{c}, T\right] .
$$

Taking the supremum over all partitions $0 \leq t_{1}<\cdots<t_{n} \leq T$ in (2.4) and (2.5) we obtain (2.2) and (2.3) respectively.

Let $0 \leq t_{1}<\cdots<t_{n} \leq T$. If $t_{n}<T_{c}$ then (2.4) and (2.5) are obvious, hence we may assume that $t_{l} \leq T_{c}<t_{l+1}$ for some $l<n$. Further, let us set

$$
\begin{aligned}
& S_{1}=\sum_{i=1}^{l-1}\left(\left|W_{t_{i+1}}-W_{t_{i}}\right|-c\right)_{+} \leq V_{\mu}^{c}\left[0, T_{c}\right], \\
& S_{2}=\sum_{i=l+1}^{n-1}\left(\left|W_{t_{i+1}}-W_{t_{i}}\right|-c\right)_{+} \leq V_{\mu}^{c}\left[T_{c}, T\right] .
\end{aligned}
$$

Since

$$
S=S_{1}+\left(\left|W_{t_{l+1}}-W_{t_{l}}\right|-c\right)_{+}+S_{2},
$$

we may assume that $\left(\left|W_{t_{l+1}}-W_{t_{l}}\right|-c\right)_{+}>0$. Hence $W_{t_{l+1}}>W_{t_{l}}+c$ or $W_{t_{l+1}}<W_{t_{l}}-c$.

Let us consider a few cases.

- $W_{t_{l+1}}>W_{t_{l}}+c$ and $W_{t_{l}} \geq W_{T_{c}}$. In this case

$$
\left(\left|W_{t_{l+1}}-W_{t_{l}}\right|-c\right)_{+} \leq\left(\left|W_{t_{l+1}}-W_{T_{c}}\right|-c\right)_{+}
$$

and we have $\left(\left|W_{t_{l+1}}-W_{T_{c}}\right|-c\right)_{+}+S_{2} \leq V_{\mu}^{c}\left[T_{c}, T\right]$ so

$$
S=S_{1}+\left(\left|W_{t_{l+1}}-W_{t_{l}}\right|-c\right)_{+}+S_{2} \leq V_{\mu}^{c}\left[0, T_{c}\right]+V_{\mu}^{c}\left[T_{c}, T\right] .
$$

- $W_{t_{l+1}}>W_{t_{l}}+c, W_{t_{l}}<W_{T_{c}}=W_{T_{\text {sup }}^{c}}-c$ and $W_{t_{l+1}} \leq W_{T_{\text {sup }}^{c}}$. In this case $t_{l}<T_{\text {sup }}^{c}$ (since for $T_{\text {sup }}^{c} \leq t \leq T_{c}, W_{t} \geq W_{T_{c}}$ ) and

$$
\left(\left|W_{t_{l+1}}-W_{t_{l}}\right|-c\right)_{+} \leq\left(\left|W_{T_{\text {sup }}^{c}}-W_{t_{l}}\right|-c\right)_{+} .
$$

Just as before, $S_{1}+\left(\left|W_{T_{\text {sup }}^{c}}-W_{t_{l}}\right|-c\right)_{+} \leq V_{\mu}^{c}\left[0, T_{c}\right]$ and

$$
S=S_{1}+\left(\left|W_{t_{l+1}}-W_{t_{l}}\right|-c\right)_{+}+S_{2} \leq V_{\mu}^{c}\left[0, T_{c}\right]+V_{\mu}^{c}\left[T_{c}, T\right] .
$$

- $W_{t_{l+1}}>W_{t_{l}}+c, W_{t_{l}}<W_{T_{c}}=W_{T_{\text {sup }}^{c}}-c$ and $W_{t_{l+1}}>W_{T_{\text {sup }}^{c}}$. In this case again $t_{l}<T_{\text {sup }}^{c}$ and

$$
\begin{aligned}
\left(\left|W_{t_{l+1}}-W_{t_{l}}\right|-c\right)_{+} & =W_{t_{l+1}}-W_{t_{l}}-c=W_{t_{l+1}}-W_{T_{c}}+W_{T_{\text {sup }}^{c}}-W_{t_{l}}-2 c \\
& =\left(\left|W_{T_{\text {sup }}^{c}}-W_{t_{l}}\right|-c\right)_{+}+\left(\left|W_{t_{l+1}}-W_{T_{c}}\right|-c\right)_{+} .
\end{aligned}
$$


Again $S_{1}+\left(\left|W_{T_{\text {sup }}^{c}}-W_{t_{l}}\right|-c\right)_{+} \leq V_{\mu}^{c}\left[0, T_{c}\right]$ as well as $\left(\left|W_{t_{l+1}}-W_{T_{c}}\right|-c\right)_{+}+S_{2}$ $\leq V_{\mu}^{c}\left[T_{c}, T\right]$, so we get

$$
S=S_{1}+\left(\left|W_{t_{l+1}}-W_{t_{l}}\right|-c\right)_{+}+S_{2} \leq V_{\mu}^{c}\left[0, T_{c}\right]+V_{\mu}^{c}\left[T_{c}, T\right] .
$$

- $W_{t_{l+1}}<W_{t_{l}}-c$. In this case $\left|W_{t_{l+1}}-W_{t_{l}}\right|>c$, hence

$$
\begin{aligned}
\left(\left|W_{t_{l+1}}-W_{t_{l}}\right|-c\right)_{+} & =\left|W_{t_{l+1}}-W_{t_{l}}\right|-c \\
& \leq\left(\left|W_{T_{c}}-W_{t_{l}}\right|-c\right)+c+\left(\left|W_{t_{l+1}}-W_{T_{c}}\right|-c\right) \\
& \leq\left(\left|W_{T_{c}}-W_{t_{l}}\right|-c\right)_{+}+c+\left(\left|W_{t_{l+1}}-W_{T_{c}}\right|-c\right)_{+} .
\end{aligned}
$$

We also have

$$
\left(\left|W_{t_{l+1}}-W_{t_{l}}\right|-c\right)_{+} \leq\left(\left|W_{T_{\text {sup }}^{c}}-\inf _{T_{c} \leq t \leq T} W_{t}\right|-c\right)_{+} .
$$

Thus we get (2.4) and (2.5), completing the proof.

We will also need the following

Lemma 3. For any $\mu \geq 0$ and $c>0$,

$$
P\left(T_{c}<\frac{1}{2} \mathbb{E} T_{c}\right) \leq \frac{7}{8}
$$

Proof. By results of Taylor (cf. [7]), $T_{c}$ has the following moment generating function:

$$
\mathbb{E} \exp \left(-\beta T_{c}\right)=\frac{\sqrt{\mu^{2}+2 \beta} \exp (-\mu c)}{\sqrt{\mu^{2}+2 \beta} \cosh \left(\sqrt{\mu^{2}+2 \beta} c\right)-\mu \sinh \left(\sqrt{\mu^{2}+2 \beta} c\right)} .
$$

From the above formula one can derive moments of $T_{c}$ :

$$
\begin{aligned}
\mathbb{E} T_{c} & = \begin{cases}\left(e^{2 \mu c}-1-2 \mu c\right) /\left(2 \mu^{2}\right) & \text { for } \mu>0 \\
c^{2} & \text { for } \mu=0\end{cases} \\
& =c^{2}\left(1+\frac{2}{3} \mu c+\frac{1}{3} \mu^{2} c^{2}+\cdots\right)
\end{aligned}
$$

and

$$
\begin{array}{rlr}
\mathbb{E} T_{c}^{2} & = \begin{cases}\left(e^{4 \mu c}-6 e^{2 \mu c} \mu c+e^{2 \mu c}+2 \mu^{2} c^{2}-2\right) /\left(2 \mu^{4}\right) & \text { for } \mu>0 \\
\frac{5}{3} c^{4} & \text { for } \mu=0\end{cases} \\
& =\frac{5}{3} c^{4}\left(1+\frac{36}{25} \mu c+\frac{94}{75} \mu^{2} c^{2}+\cdots\right) .
\end{array}
$$

By the Paley-Zygmund inequality, for $\lambda \in(0,1)$,

$$
\mathbb{P}\left(T_{c} \geq \lambda \mathbb{E} T_{c}\right) \geq(1-\lambda)^{2} \frac{\left(\mathbb{E} T_{c}\right)^{2}}{\mathbb{E} T_{c}^{2}} .
$$

For $\mu=0$ we have $\left(\mathbb{E} T_{c}\right)^{2} / \mathbb{E} T_{c}^{2}=3 / 5$, and for $\mu>0$ with standard calculus 
one can show that

$$
\frac{\left(\mathbb{E} T_{c}\right)^{2}}{\mathbb{E} T_{c}^{2}}=\frac{1}{2} \frac{\left(e^{2 \mu c}-1-2 \mu c\right)^{2}}{e^{4 \mu c}-6 e^{2 \mu c} \mu c+e^{2 \mu c}+2 \mu^{2} c^{2}-2} \geq \frac{1}{2} .
$$

Finally, from the above inequalities, for $\lambda=1 / 2$ we obtain

$$
\mathbb{P}\left(T_{c}<\frac{1}{2} \mathbb{E} T_{c}\right)=1-\mathbb{P}\left(T_{c} \geq \frac{1}{2} \mathbb{E} T_{c}\right) \leq 1-\frac{1}{4} \frac{1}{2}=\frac{7}{8} .
$$

Now we are ready to prove estimates of $\mathbb{E} V_{\mu}^{c}([0, T])$ for $T$ comparable with $\mathbb{E} T_{c}$. We have

Theorem 1. For any $T \geq \frac{1}{2} \mathbb{E} T_{c}$,

$$
\frac{1}{120}\left(\frac{c}{\mathbb{E} T_{c}}+\mu\right) T \leq \mathbb{E} V_{\mu}^{c}([0, T]) \leq 64\left(\frac{c}{\mathbb{E} T_{c}}+\mu\right) T .
$$

Proof. First we estimate $\mathbb{E} V_{\mu}^{c}[0, T]$ from above. Let us observe that $V_{\mu}^{c}\left[0, T_{c}\right]=\left(W_{T_{\text {sup }}^{c}}-W_{T_{\text {inf }}^{c}}-c\right)_{+} \leq W_{T_{\text {sup }}^{c}}$, since $W_{T_{\text {inf }}^{c}} \geq-c$. Now, from this, (2.2) and independence of $\left(W_{t}-W_{T_{c}}, t \geq T_{c}\right)$ and $T_{c}$ it follows that

$$
\begin{aligned}
\mathbb{E} V_{\mu}^{c}([0, T]) \leq & \mathbb{E} W_{T_{\text {sup }}^{c}}+c+\mathbb{E}\left[V_{\mu}^{c}\left[T_{c}, T\right] ; T_{c}<\frac{1}{2} \mathbb{E} T_{c}\right] \\
& +\mathbb{E}\left[V_{\mu}^{c}\left[T_{c}, T\right] ; T_{c} \geq \frac{1}{2} \mathbb{E} T_{c}\right] \\
\leq & \mathbb{E} W_{T_{\text {sup }}^{c}}+c+\mathbb{E} V_{\mu}^{c}[0, T] \cdot \mathbb{P}\left(T_{c}<\frac{1}{2} \mathbb{E} T_{c}\right) \\
& +\mathbb{E} V_{\mu}^{c}\left[\frac{1}{2} \mathbb{E} T_{c}, T\right] \cdot \mathbb{P}\left(T_{c} \geq \frac{1}{2} \mathbb{E} T_{c}\right) .
\end{aligned}
$$

Since $\mathbb{E} W_{T_{\text {sup }}^{c}}=c+\mu \mathbb{E} T_{c} \geq c($ cf. [7]), the last inequality and Lemma 3 give

$$
\begin{aligned}
\mathbb{E} V_{\mu}^{c}([0, T]) & \leq \frac{\mathbb{E} W_{T_{\text {sup }}^{c}}+c}{\mathbb{P}\left(T_{c} \geq \frac{1}{2} \mathbb{E} T_{c}\right)}+\mathbb{E} V_{\mu}^{c}\left[\frac{1}{2} \mathbb{E} T_{c}, T\right] \\
& \leq \frac{2 \mathbb{E} W_{T_{\text {sup }}^{c}}}{1 / 8}+\mathbb{E} V_{\mu}^{c}\left[\frac{1}{2} \mathbb{E} T_{c}, T\right] \\
& \leq 16 \mathbb{E} W_{T_{\text {sup }}^{c}}+\mathbb{E} V_{\mu}^{c}\left[\frac{1}{2} \mathbb{E} T_{c}, T\right] .
\end{aligned}
$$

Applying shift invariance of $V_{\mu}^{c}$ and iterating this inequality $\left\lfloor 2 T / \mathbb{E} T_{c}\right\rfloor$ times we get $\mathbb{E} V_{\mu}^{c}([0, T]) \leq 16 \mathbb{E} W_{T_{\text {sup }}^{c}} \cdot\left(\left\lfloor 2 T / \mathbb{E} T_{c}\right\rfloor+1\right)$. Applying the identity $\mathbb{E} W_{T_{\text {sup }}}=c+\mu \mathbb{E} T_{c}$ and the inequality $\left\lfloor 2 T / \mathbb{E} T_{c}\right\rfloor \geq 1$ we finally obtain

$$
\begin{aligned}
\mathbb{E} V_{\mu}^{c}([0, T]) & \leq 16 \mathbb{E} W_{T_{\text {sup }}^{c}} \cdot\left(\left\lfloor 2 T / \mathbb{E} T_{c}\right\rfloor+1\right) \\
& \leq 16\left(c+\mu \mathbb{E} T_{c}\right) \frac{4 T}{\mathbb{E} T_{c}} \leq 64\left(\frac{c}{\mathbb{E} T_{c}}+\mu\right) T .
\end{aligned}
$$


In order to get an estimate from below let us divide $[0, T]$ into $\left\lfloor 2 T / \mathbb{E} T_{c}\right\rfloor$ intervals of length $\frac{1}{2} \mathbb{E} T_{c}:[0, T]=\left[0, \frac{1}{2} \mathbb{E} T_{c}\right] \cup\left[\frac{1}{2} \mathbb{E} T_{c}, \mathbb{E} T_{c}\right] \cup \cdots \cup\left[\left\lfloor 2 T / \mathbb{E} T_{c}\right\rfloor\right.$. $\left.\mathbb{E} T_{c}, T\right]$. Let $a_{j}=(j / 2) \mathbb{E} T_{c}, j=0,1, \ldots,\left\lfloor 2 T / \mathbb{E} T_{c}\right\rfloor, \Delta T=\frac{1}{2} \mathbb{E} T_{c} \geq \frac{1}{2} c^{2}$ and $y=\frac{3}{2} c+\mu \Delta T$. We have

$$
\begin{aligned}
\mathbb{E} V_{\mu}^{c}([0, T]) & \geq \sum_{j=0}^{\left\lfloor 2 T / \mathbb{E} T_{c}\right\rfloor-1} \mathbb{E} V_{\mu}^{c}\left(\left[a_{j}, a_{j}+\Delta T\right]\right) \\
& \geq \sum_{j=0}^{\left\lfloor 2 T / \mathbb{E} T_{c}\right\rfloor-1} \mathbb{E}\left(\sup _{0 \leq s \leq \Delta T} W_{a_{j}+s}-W_{a_{j}}-c\right)_{+} \\
& \geq\left\lfloor 2 T / \mathbb{E} T_{c}\right\rfloor(y-c) \mathbb{P}\left(\sup _{0 \leq s \leq \Delta T} W_{s} \geq y\right) .
\end{aligned}
$$

For the process $\left(W_{t}, t \geq 0\right)$ we have

$$
\begin{aligned}
\mathbb{P}\left(\sup _{0 \leq s \leq \Delta T} W_{s} \geq y\right) & \geq \mathbb{P}\left(W_{\Delta T} \geq y\right)=\mathbb{P}\left(B_{\Delta T} \geq y-\mu \Delta T\right) \\
& =\frac{1}{2} \operatorname{Erfc}\left(\frac{y-\mu \Delta T}{\sqrt{2 \Delta T}}\right),
\end{aligned}
$$

where $\operatorname{Erfc}(x)$ is the complementary error function, $\operatorname{Erfc}(x)=\frac{2}{\sqrt{\pi}} \int_{x}^{\infty} e^{-t^{2}} d t$. Since $\Delta T \geq \frac{1}{2} c^{2}$ we have

$$
\begin{aligned}
\mathbb{P}\left(\sup _{0 \leq s \leq \Delta T} W_{s} \geq y\right) & \geq \frac{1}{2} \operatorname{Erfc}\left(\frac{\frac{3}{2} c+\mu \Delta T-\mu \Delta T}{\sqrt{c^{2}}}\right) \\
& =\frac{1}{2} \operatorname{Erfc}\left(\frac{3}{2}\right) .
\end{aligned}
$$

Now, from (2.6) and (2.8) we get the estimate from below

$$
\begin{aligned}
\mathbb{E} V_{\mu}^{c}([0, T]) & \geq\left\lfloor 2 T / \mathbb{E} T_{c}\right\rfloor(y-c) \mathbb{P}\left(\sup _{0 \leq s \leq \Delta T} W_{s} \geq y\right) \\
& \geq \frac{T}{\mathbb{E} T_{c}}\left(\frac{3}{2} c+\frac{1}{2} \mu \mathbb{E} T_{c}-c\right) \frac{1}{2} \operatorname{Erfc}\left(\frac{3}{2}\right) \\
& =\frac{1}{4} \operatorname{Erfc}\left(\frac{3}{2}\right)\left(\frac{c}{\mathbb{E} T_{c}}+\mu\right) T \geq \frac{1}{120}\left(\frac{c}{\mathbb{E} T_{c}}+\mu\right) T .
\end{aligned}
$$

Corollary 1. For $T \geq \frac{1}{2} \mathbb{E} T_{c}$,

$$
\frac{1}{264}\left(\frac{1}{c}+\mu\right) T \leq \mathbb{E} V_{\mu}^{c}[0, T] \leq 64\left(\frac{1}{c}+\mu\right) T .
$$

Proof. The upper bound follows immediately from Theorem 1 and the inequality

$$
\mathbb{E} T_{c}=\frac{e^{2 \mu c}-1-2 \mu c}{2 \mu^{2}}=c^{2}\left(1+\frac{2}{3} \mu c+\frac{1}{3} \mu^{2} c^{2}+\ldots\right) \geq c^{2} .
$$

In order to prove the lower bound let us consider two cases. 
- $\mu c \geq 1$. In this case we have $1 / c \leq \mu$ and, by Theorem 1 ,

$$
\begin{aligned}
\mathbb{E} V_{\mu}^{c}[0, T] & \geq \frac{1}{120} \mu T \geq \frac{1}{120}\left(\frac{1}{2} \frac{1}{c}+\frac{1}{2} \mu\right) T \\
& =\frac{1}{240}\left(\frac{1}{c}+\mu\right) T .
\end{aligned}
$$

- $\mu c<1$. In this case, since $\mathbb{E} T_{c} / c^{2}$ is an increasing function of $\mu c$, we have $\mathbb{E} T_{c} / c^{2} \leq\left(e^{2}-1-2\right) / 2<2.2$. Thus $c / \mathbb{E} T_{c} \geq 1 / 2.2 c$, and by Theorem 1 ,

$$
\begin{aligned}
\mathbb{E} V_{\mu}^{c}[0, T] & \geq \frac{1}{120}\left(\frac{1}{2.2 c}+\mu\right) T \geq \frac{1}{120}\left(\frac{1}{2.2 c}+\frac{1}{2.2} \mu\right) T \\
& =\frac{1}{264}\left(\frac{1}{c}+\mu\right) T .
\end{aligned}
$$

3. Estimates for short time intervals. In order to prove estimates of $V_{\mu}^{c}([0, T])$ for small $T$ s (smaller than $\left.\frac{1}{2} \mathbb{E} T_{c}\right)$ we will need two more lemmas.

Lemma 4. For any $T \leq \frac{1}{2} \mathbb{E} T_{c}$,

$$
\begin{aligned}
\mathbb{E}\left(\sup _{0 \leq t \leq T} W_{t}-\inf _{0 \leq t \leq T} W_{t}-c\right)_{+} & \leq \mathbb{E} V_{\mu}^{c}([0, T]) \\
& \leq 16 \mathbb{E}\left(\sup _{0 \leq t \leq T} W_{t}-\inf _{0 \leq t \leq T} W_{t}-c\right)_{+} .
\end{aligned}
$$

Proof. The estimate from below is self-evident. In order to obtain the estimate from above we apply Lemma 1, Lemma 2, independence of $\left(W_{t}-W_{T_{c}}, t \geq T_{c}\right)$ and $T_{c}$, and Lemma 3 :

$$
\begin{aligned}
\mathbb{E} V_{\mu}^{c}([0, T]) \leq & \mathbb{E}\left(W_{T_{\text {sup }}^{c}}-W_{T_{\text {inf }}^{c}}-c\right)_{+} \\
& +\mathbb{E}\left(W_{T_{\text {sup }}^{c}}-\inf _{T_{c} \leq t \leq T} W_{t}-c\right)_{+} \\
& +\mathbb{E}\left[V_{\mu}^{c}\left[T_{c}, T\right] ; T_{c}<T\right] \\
\leq & 2 \mathbb{E}\left(\sup _{0 \leq t \leq T} W_{t}-\inf _{0 \leq t \leq T} W_{t}-c\right)_{+} \\
& +\mathbb{E} V_{\mu}^{c}\left[T_{c}, T\right] \cdot \mathbb{P}\left(T_{c}<\frac{1}{2} \mathbb{E} T_{c}\right) \\
\leq & 2 \mathbb{E}\left(\sup _{0 \leq t \leq T} W_{t}-\inf _{0 \leq t \leq T} W_{t}-c\right)_{+}+\frac{7}{8} \mathbb{E} V_{\mu}^{c}[0, T] .
\end{aligned}
$$

Thus we get

$$
\mathbb{E} V_{\mu}^{c}([0, T]) \leq 16 \mathbb{E}\left(\sup _{0 \leq t \leq T} W_{t}-\inf _{0 \leq t \leq T} W_{t}-c\right)_{+} .
$$


Lemma 5. If $\sqrt{T}+\mu T \geq c$, then

$$
\begin{aligned}
\frac{1}{44}(2 \sqrt{T}+\mu T-c) & \leq \mathbb{E}\left(\sup _{0 \leq t \leq T} W_{t}-\inf _{0 \leq t \leq T} W_{t}-c\right)_{+} \\
& \leq 1.6(2 \sqrt{T}+\mu T-c),
\end{aligned}
$$

and if $\sqrt{T}+\mu T<c$, then

$$
\begin{aligned}
\frac{1}{12} T^{3 / 2} \frac{e^{-(c-\mu T)^{2} /(2 T)}}{(c-\mu T)^{2}} & \leq \mathbb{E}\left(\sup _{0 \leq t \leq T} W_{t}-\inf _{0 \leq t \leq T} W_{t}-c\right)_{+} \\
& \leq 3.2 T^{3 / 2} \frac{e^{-(c-\mu T)^{2} /(2 T)}}{(c-\mu T)^{2}}
\end{aligned}
$$

Proof. Let us first consider the case $\sqrt{T}+\mu T \geq c$. We have

$$
\begin{aligned}
\mathbb{E}\left(\sup _{0 \leq t \leq T} W_{t}-\inf _{0 \leq t \leq T} W_{t}\right. & -c)_{+} \\
& \leq \mathbb{E}\left(\sup _{0 \leq t \leq T} B_{t}-\inf _{0 \leq t \leq T} B_{t}+\sqrt{T}+\mu T-c\right)_{+} \\
& =\mathbb{E}\left(\sup _{0 \leq t \leq T} B_{t}-\inf _{0 \leq t \leq T} B_{t}+\sqrt{T}+\mu t-c\right) \\
& =(\sqrt{8 / \pi}+1) \sqrt{T}+\mu T-c \\
& \leq 1.6(2 \sqrt{T}+\mu t-c) .
\end{aligned}
$$

In order to get the estimate from below we apply formula (2.7). We have $\inf _{0 \leq t \leq T} W_{t} \leq W_{0}=0$, so that

$$
\begin{aligned}
\mathbb{E}\left(\sup _{0 \leq t \leq T} W_{t}-\inf _{0 \leq t \leq T}\right. & \left.W_{t}-c\right)_{+} \\
& \geq(2 \sqrt{T}+\mu T-c) \mathbb{P}\left(\sup _{0 \leq t \leq T} W_{s} \geq 2 \sqrt{T}+\mu T\right) \\
& \geq(2 \sqrt{T}+\mu T-c) \frac{1}{2} \operatorname{Erfc}\left(\frac{2 \sqrt{T}+\mu T-\mu T}{\sqrt{2 T}}\right) \\
& =(2 \sqrt{T}+\mu T-c) \frac{1}{2} \operatorname{Erfc}(\sqrt{2}) \\
& \geq \frac{1}{44}(2 \sqrt{T}+\mu T-c) .
\end{aligned}
$$

In the case $\sqrt{T}+\mu T<c$ we have to apply more exact formulae. For the estimate from below we calculate

$$
\begin{aligned}
\mathbb{E}\left(\sup _{0 \leq t \leq T} W_{t}-\inf _{0 \leq t \leq T} W_{t}-c\right)_{+} & \geq \mathbb{E}\left(\sup _{0 \leq t \leq T} W_{t}-c\right)_{+} \\
& =\int_{c}^{\infty} \mathbb{P}\left(\sup _{0 \leq t \leq T} W_{s} \geq y\right) d y \\
& \geq \frac{1}{2} \int_{c}^{\infty} \operatorname{Erfc}\left(\frac{y-\mu T}{\sqrt{2 T}}\right) d y .
\end{aligned}
$$


For the estimate from above we use the following formula valid for a standard Brownian motion $\left(B_{t}, t \geq 0\right)$ and $y \geq 0$ (cf. [1] or [3]):

$$
\mathbb{P}\left(\sup _{0 \leq t \leq T} B_{t}-\inf _{0 \leq t \leq T} B_{t} \geq y\right)=4 \sum_{k=1}^{\infty}(-1)^{k+1} k \operatorname{Erfc}\left(\frac{k y}{\sqrt{2 T}}\right) .
$$

For $k \geq 1$ and $y \geq \sqrt{T}$ we have

$$
\begin{aligned}
\operatorname{Erfc}\left(\frac{(2 k+1) y}{\sqrt{2 T}}\right) & =\frac{2}{\sqrt{\pi}} \int_{(2 k+1) y / \sqrt{2 T}}^{\infty} e^{-t^{2}} d t \\
& =\frac{2}{\sqrt{\pi}} \int_{2 k y / \sqrt{2 T}}^{\infty} e^{-(t+y / \sqrt{2 T})^{2}} d t \\
& \leq \frac{2}{\sqrt{\pi}} \int_{2 k y / \sqrt{2 T}}^{\infty} e^{-t^{2}-2 k} d t \\
& \leq \frac{2 k}{2 k+1} \operatorname{Erfc}\left(\frac{2 k y}{\sqrt{2 T}}\right),
\end{aligned}
$$

hence

$$
\mathbb{P}\left(\sup _{0 \leq t \leq T} B_{t}-\inf _{0 \leq t \leq T} B_{t} \geq y\right) \leq 4 \operatorname{Erfc}\left(\frac{y}{\sqrt{2 T}}\right)
$$

and

$$
\begin{aligned}
\mathbb{E}\left(\sup _{0 \leq t \leq T} W_{t}-\inf _{0 \leq t \leq T} W_{t}-c\right)_{+} & \leq \mathbb{E}\left(\sup _{0 \leq t \leq T} B_{t}-\inf _{0 \leq t \leq T} B_{t}+\mu T-c\right)_{+} \\
& =\int_{c-\mu T}^{\infty} \mathbb{P}\left(\sup _{0 \leq t \leq T} B_{t}-\inf _{0 \leq t \leq T} B_{t} \geq y\right) d y \\
& \leq 4 \int_{c-\mu T}^{\infty} \operatorname{Erfc}\left(\frac{y}{\sqrt{2 T}}\right) d y \\
& =4 \int_{c}^{\infty} \operatorname{Erfc}\left(\frac{y-\mu T}{\sqrt{2 T}}\right) d y .
\end{aligned}
$$

The last step is to estimate

$$
\int_{c}^{\infty} \operatorname{Erfc}\left(\frac{y-\mu T}{\sqrt{2 T}}\right) d y=\sqrt{2 T} \int_{(c-\mu T) / \sqrt{2 T}}^{\infty} \operatorname{Erfc}(z) d z
$$

for $c \geq \mu T+\sqrt{T}$. The known estimate

$$
\frac{2}{\sqrt{\pi}} \frac{e^{-d^{2}}}{d+\sqrt{d^{2}+2}}<\operatorname{Erfc}(d) \leq \frac{2}{\sqrt{\pi}} \frac{e^{-d^{2}}}{d+\sqrt{d^{2}+\pi / 4}}
$$


for $d \geq 0$ (cf. [5]) and the equality $\int_{d}^{\infty} \operatorname{Erfc}(z) d z=e^{-d^{2}} / \sqrt{\pi}-d \operatorname{Erfc}(d)$ for $d \geq 1 / \sqrt{2}$ yield

$$
\begin{aligned}
\int_{d}^{\infty} \operatorname{Erfc}(z) d z & \geq \frac{e^{-d^{2}}}{\sqrt{\pi}}-\frac{2}{\sqrt{\pi}} \frac{e^{-d^{2} d}}{d+\sqrt{d^{2}+\pi / 4}} \\
& =\frac{e^{-d^{2}}}{d^{2}} \frac{\sqrt{\pi}}{4\left(1+\sqrt{1+\pi /\left(4 d^{2}\right)}\right)^{2}} \geq \frac{1}{16} \frac{e^{-d^{2}}}{d^{2}}
\end{aligned}
$$

and

$$
\begin{aligned}
\int_{d}^{\infty} \operatorname{Erfc}(z) d z & \leq \frac{e^{-d^{2}}}{\sqrt{\pi}}-\frac{2}{\sqrt{\pi}} \frac{e^{-d^{2}} d}{d+\sqrt{d^{2}+2}} \\
& =\frac{e^{-d^{2}}}{d^{2}} \frac{2}{\sqrt{\pi}\left(1+\sqrt{1+2 / d^{2}}\right)^{2}} \leq \frac{1}{2 \sqrt{\pi}} \frac{e^{-d^{2}}}{d^{2}} .
\end{aligned}
$$

Putting together the above inequalities for $d=(c-\mu T) / \sqrt{2 T}>1 / \sqrt{2}$ we finally get the assertion.

REMARK 1. In the proof above we could have tried to use the exact formula for $\mathbb{E}\left(\sup _{0 \leq t \leq T} W_{t}-\inf _{0 \leq t \leq T} W_{t}-c\right)_{+}$, since the formula for $\mathbb{P}\left(\sup _{0 \leq t \leq T} W_{t}-\inf _{0 \leq t \leq T} W_{t} \geq y\right)$ is known (cf. [1], [2] or [6]); however, we preferred to avoid this because the latter formula seems much more complicated than the one for $B_{t}$.

Lemmas 4 and 5 immediately yield

Theorem 2. If $c-\mu T \leq \sqrt{T}<\sqrt{\frac{1}{2} \mathbb{E} T_{c}}$ then

$$
\frac{1}{44}(2 \sqrt{T}+\mu T-c) \leq \mathbb{E} V_{\mu}^{c}([0, T]) \leq 26(2 \sqrt{T}+\mu T-c),
$$

and if $\sqrt{T}<\min \left\{c-\mu T, \sqrt{\frac{1}{2} \mathbb{E} T_{c}}\right\}$ then

$$
\frac{1}{12} T^{3 / 2} \frac{e^{-(c-\mu T)^{2} /(2 T)}}{(c-\mu T)^{2}} \leq \mathbb{E} V_{\mu}^{c}([0, T]) \leq 52 T^{3 / 2} \frac{e^{-(c-\mu T)^{2} /(2 T)}}{(c-\mu T)^{2}} .
$$

Combining Corollary 1 and Theorem 2 we get

Corollary 2. Set $\chi(c, \mu)=\sqrt{\mathbb{E} T_{c}}=c \sqrt{1+\frac{2}{3} \mu c+\frac{1}{3} \mu^{2} c^{2}+\cdots}$.

- If $\sqrt{T} \geq \chi(c, \mu)$, then

$$
\frac{1}{264}\left(\frac{1}{c}+\mu\right) T \leq \mathbb{E} V_{\mu}^{c}[0, T] \leq 64\left(\frac{1}{c}+\mu\right) T .
$$

- If $c-\mu T \leq \sqrt{T}<\chi(c, \mu)$, then

$$
\frac{1}{747}(2 \sqrt{T}+\mu T-c) \leq \mathbb{E} V_{\mu}^{c}[0, T] \leq 340(2 \sqrt{T}+\mu T-c) .
$$


- If $\sqrt{T}<c-\mu T$, then

$$
\frac{1}{227} T^{3 / 2} \frac{e^{-(c-\mu T)^{2} /(2 T)}}{(c-\mu T)^{2}} \leq \mathbb{E} V_{\mu}^{c}[0, T] \leq 493 T^{3 / 2} \frac{e^{-(c-\mu T)^{2} /(2 T)}}{(c-\mu T)^{2}} .
$$

Proof. The estimates for $\sqrt{T} \geq \chi(c, \mu)$ follow from Corollary 1. By Theorem 2 we need only prove that if $\max \{c-\mu T, \chi(c, \mu) / \sqrt{2}\} \leq \sqrt{T} \leq \chi(c, \mu)$, then

$$
\frac{1}{747}(2 \sqrt{T}+\mu T-c) \leq \mathbb{E} V_{\mu}^{c}[0, T] \leq 340(2 \sqrt{T}+\mu T-c),
$$

and if $\chi(c, \mu) / \sqrt{2} \leq \sqrt{T}<c-\mu T$, then

$$
\frac{1}{227} T^{3 / 2} \frac{e^{-(c-\mu T)^{2} /(2 T)}}{(c-\mu T)^{2}} \leq \mathbb{E} V_{\mu}^{c}[0, T] \leq 493 T^{3 / 2} \frac{e^{-(c-\mu T)^{2} /(2 T)}}{(c-\mu T)^{2}} .
$$

In order to prove the lower bound in (3.1) let us notice that for $T \geq$ $\chi^{2}(c, \mu) / 2 \geq c^{2} / 2$ we have $1 / c \geq 1 / \sqrt{2 T}$, and by Corollary 1 ,

$$
\begin{aligned}
\mathbb{E} V_{\mu}^{c}[0, T] & \geq \frac{1}{264}\left(\frac{1}{c}+\mu\right) T \geq \frac{1}{264}\left(\frac{1}{\sqrt{2 T}}+\mu\right) T \\
& =\frac{1}{264}\left(\frac{1}{\sqrt{2}} \sqrt{T}+\mu T\right) \geq \frac{1}{747}(2 \sqrt{T}+\mu T-c) .
\end{aligned}
$$

To prove the upper bound we consider two cases.

- $\mu c \geq 1$. In this case $1 / c \leq \mu$. By Corollary 1 , since $\sqrt{2} \sqrt{T} \geq c$ we have

$$
\mathbb{E} V_{\mu}^{c}[0, T] \leq \frac{1}{64}\left(\frac{1}{c}+\mu\right) T \leq \frac{1}{32} \mu T \leq \frac{1}{32}(2 \sqrt{T}+\mu T-c) .
$$

- $\mu c<1$. In this case, since $\chi^{2}(c, \mu) / c^{2}=\left(e^{2 \mu c}-1-2 \mu c\right) /\left(2 \mu^{2} c^{2}\right)$ is an increasing function of $\mu c$ and $T \leq \chi^{2}(c, \mu)$ we have

$$
\frac{T}{c} \leq c \frac{\chi^{2}(c, \mu)}{c^{2}} \leq c \frac{e^{2}-1-2}{2}<2.2 c .
$$

Now, by Corollary 1 and the inequality $c \leq \sqrt{2} \sqrt{T}$,

$$
\begin{aligned}
\mathbb{E} V_{\mu}^{c}[0, T] & \leq 64\left(\frac{1}{c}+\mu\right) T \leq 64(2.2 c+\mu T) \\
& \leq 64(2.2 \sqrt{2} \sqrt{T}+\mu T) \\
& \leq \frac{64 \cdot 2.2 \sqrt{2}}{2-\sqrt{2}}((2-\sqrt{2}) \sqrt{T}+\mu T+\sqrt{2} \sqrt{T}-c) \\
& \leq 340(2 \sqrt{T}+\mu T-c) .
\end{aligned}
$$

Now let us prove (3.2). Again, for $T \geq \chi^{2}(c, \mu) / 2$ we have $1 / c \geq 1 / \sqrt{2 T}$, and by Corollary 1 and the inequality $\sqrt{T}<c-\mu T$ we have $e^{-(c-\mu T)^{2} /(2 T)} \leq$ 
$e^{-1 / 2}$, so

$$
\begin{aligned}
\mathbb{E} V_{\mu}^{c}[0, T] & \geq \frac{1}{264}\left(\frac{1}{c}+\mu\right) T \geq \frac{1}{264}\left(\frac{1}{\sqrt{2 T}}+\mu\right) T \\
& \geq \frac{1}{264 \sqrt{2}} \sqrt{T} \geq \frac{1}{264 \sqrt{2} e^{-1 / 2}} T^{3 / 2} \frac{e^{-(c-\mu T)^{2} /(2 T)}}{(c-\mu T)^{2}} \\
& \geq \frac{1}{227} T^{3 / 2} \frac{e^{-(c-\mu T)^{2} /(2 T)}}{(c-\mu T)^{2}} .
\end{aligned}
$$

In order to prove the upper bound let us observe that $c / \sqrt{2} \leq \sqrt{T}<c-\mu T$, so $(c-\mu T) / \sqrt{T} \leq \sqrt{2}, T / c \leq \sqrt{T}$ and $\mu T<(1-1 / \sqrt{2}) c$. By Corollary 1 ,

$$
\begin{aligned}
\mathbb{E} V_{\mu}^{c}[0, T] & \leq 64\left(\frac{1}{c}+\mu\right) T \leq 64(\sqrt{T}+(1-1 / \sqrt{2}) c) \\
& \leq 64(\sqrt{T}+(1-1 / \sqrt{2}) \sqrt{2} \sqrt{T}) \\
& =64 \sqrt{2} \sqrt{T} \leq 64 \sqrt{2} \sqrt{T} 2 e \frac{e^{-(c-\mu T)^{2} /(2 T)}}{((c-\mu T) / \sqrt{T})^{2}} \\
& \leq 493 T^{3 / 2} \frac{e^{-(c-\mu T)^{2} /(2 T)}}{(c-\mu T)^{2}} .
\end{aligned}
$$

4. Appendix. Now we will explain how truncated variation appears in the upper bound of return from trading a single asset in a geometric Brownian motion model. Let us assume that the dynamics of the prices $P_{t}$ of some financial asset (e.g. stock) is $P_{t}=\exp \left(\mu t+\sigma B_{t}\right)$. We are interested in the maximal possible profit coming from trading this single instrument during the time interval $[0, T]$. This means that we buy the instrument at times $0 \leq t_{b_{1}}<\cdots<t_{b_{n}}<T$ and sell it at times $t_{s_{1}}<\cdots<t_{s_{n}} \leq T$, such that $t_{b_{1}}<t_{s_{1}}<t_{b_{2}}<t_{s_{2}}<\cdots<t_{b_{n}}<t_{s_{n}}$, in order to obtain the maximal possible profit.

Furthermore, we assume that for every transaction we have to pay a flat commission and $\gamma$ is the fraction of the transaction value paid for the commission.

The maximal possible rate of return from our strategy is

$$
\sup _{n} \sup _{0 \leq t_{b_{1}}<t_{s_{1}}<\cdots<t_{b_{n}}<t_{s_{n}} \leq T} \frac{P_{t_{s_{1}}}}{P_{t_{b_{1}}}} \frac{1-\gamma}{1+\gamma} \cdots \frac{P_{t_{s_{n}}}}{P_{t_{b_{n}}}} \frac{1-\gamma}{1+\gamma}-1 .
$$

Indeed, if at time $t_{b_{1}}$ we buy e.g. $n_{1}$ stocks for $P_{t_{b_{1}}}$, then we have to invest $n_{1} \cdot P_{t_{b_{1}}} \cdot(1+\gamma)$. At time $t_{s_{1}}$ we sell $n_{1}$ stocks and after paying commission we obtain $n_{1} \cdot P_{t_{s_{1}}} \cdot(1-\gamma)$. The rate of return from these two tradings equals $\frac{P_{t_{s_{1}}}}{P_{t_{b_{1}}}} \frac{1-\gamma}{1+\gamma}-1$. We again invest the money obtained and after $n$ transactions 
we get the rate of return

$$
\prod_{i=1}^{n}\left\{\frac{P_{t_{s_{i}}}}{P_{t_{b_{i}}}} \frac{1-\gamma}{1+\gamma}\right\}-1
$$

Let $M_{n}$ be the set of all partitions

$$
\pi=\left\{0 \leq t_{b_{1}}<t_{s_{1}}<\cdots<t_{b_{n}}<t_{s_{n}} \leq T\right\} .
$$

To see that $\exp \left(\sigma V_{\mu / \sigma}^{c / \sigma}[0, T]\right)-1$ with $c=\ln \frac{1+\gamma}{1-\gamma}$ is an upper bound for the rate of return let us compute

$$
\begin{aligned}
\sup _{n} \sup _{M_{n}} \prod_{i=1}^{n}\left\{\frac{P_{t_{s_{i}}}}{P_{t_{b_{i}}}} \frac{1-\gamma}{1+\gamma}\right\}=\sup _{n} \sup _{M_{n}} \prod_{i=1}^{n}\left\{\frac{\exp \left(\mu t_{s_{i}}+\sigma B_{t_{s_{i}}}\right)}{\exp \left(\mu t_{b_{i}}+\sigma B_{t_{b_{i}}}\right)} e^{-c}\right\} \\
\quad=\sup _{n} \sup _{M_{n}} \exp \left(\sigma \sum_{i=1}^{n}\left\{\left(\frac{\mu}{\sigma} t_{s_{i}}+B_{t_{s_{i}}}\right)-\left(\frac{\mu}{\sigma} t_{b_{i}}+B_{t_{b_{i}}}\right)-\frac{c}{\sigma}\right\}\right) \\
=\exp \left(\sigma \sup _{n} \sup _{n} \sum_{i=1}^{n}\left\{\left(\frac{\mu}{\sigma} t_{s_{i}}+B_{t_{s_{i}}}\right)-\left(\frac{\mu}{\sigma} t_{b_{i}}+B_{t_{b_{i}}}\right)-\frac{c}{\sigma}\right\}\right) \\
\leq \exp \left(\sigma V_{\mu / \sigma}^{c / \sigma}[0, T]\right) .
\end{aligned}
$$

This gives the bound claimed.

REMARK 2. We have proved that the maximal possible rate of return is bounded by the exponential moment of the truncated variation with the appropriate truncation level $c$. It is possible to prove, using similar techniques to the proof of Theorem 1, that the exponential moment of the truncated variation is finite. However, no bounds for the exponential moment and even for moments of order greater than one are known to the author.

Acknowledgements. I would like to express my gratitude to Prof. Krzysztof Oleszkiewicz for reading the first draft of this paper and for encouragement to submit it.

\section{References}

[1] A. N. Borodin and P. Salminen, Handbook of Brownian Motion. Facts and Formulae, Probab. Appl., Birkhäuser, Basel, 1996.

[2] K. S. Chong, R. Cowan and L. Horst, The ruin problem and cover times of asymmetric random walks and Brownian motions, Adv. Appl. Probab. 32 (2000), 177-192.

[3] W. Feller, The asymptotic distribution of the range of sums of independent random variables, Ann. Math. Statist. 22 (1952), 427-432.

[4] P. Lévy, Le mouvement brownien plane, Amer. J. Math. 62 (1940), 487-550.

[5] H. O. Pollak, A remark on "Elementary inequalities for Mills' ratio" by Yûsaku Komatu, Rep. Statist. Appl. Res. Un. Jap. Sci. Engrs. 4 (1955-57), 110.

[6] E. Tanré and P. Vallois, Range of Brownian motion with drift, J. Theoret. Probab. 19 (2006), 45-69. 
[7] H. M. Taylor, A stopped Brownian motion formula, Ann. Probab. 3 (1975), 234-246.

Rafał Łochowski

Department of Mathematical Economics

Warsaw School of Economics

Al. Niepodległości 162

02-554 Warszawa, Poland

E-mail: rlocho314@gmail.com

Received November 4, 2008;

received in final form January 12, 2009 